\title{
Reacciones adversas a los fármacos antituberculosis en pacientes con esquemas mixtos
}

\author{
Tania María Díaz Covarrubias-López,* Rafael Laniado-Laborín* $\bowtie$ \\ *Universidad Autónoma BC Valle de las Palmas; ‘niversidad Autónoma de Baja California, \\ Hospital General Tijuana, ISESALUD, SNI II, CONACYT. \\ Trabajo recibido: 03-IX-2015; aceptado: 18-II-2016
}

\begin{abstract}
RESUMEN. Antecedentes: La frecuencia real de los efectos adversos (EA) atribuibles al tratamiento de la tuberculosis resistente a los fármacos se desconoce debido a que con frecuencia no son notificados y, por ende, no se reportan. Material y métodos: Con el propósito de determinar la frecuencia de los EA en pacientes con tuberculosis sometidos a esquemas de tratamiento mixtos con fármacos de primera y segunda línea, se llevó a cabo una revisión de los archivos de la Clínica de Tuberculosis Resistente a Fármacos del Hospital General Tijuana. Resultados: Las reacciones adversas a los fármacos antituberculosis (RAFA) más frecuentes fueron la alteración de la función renal (75.9\%), hepática (82.8\%), hematológica (79.3\%), digestiva (40\%), auditiva (26.7\%), vestibular (15\%) y musculotendinosa (30\%). Conclusiones: Los EA de los fármacos antituberculosis son muy frecuentes y constituyen un factor muy importante en la pérdida de adherencia al régimen. Deben ser monitoreados activamente y mitigados con tratamiento sintomático, cambios en los horarios de administración y, si el caso lo amerita, la suspensión del fármaco en cuestión.
\end{abstract}

Palabras clave: Tuberculosis, fármacos, reacciones adversas, toxicidad.

ABSTRACT. Background: The true frequency of adverse reactions (AR) attributed to antituberculosis drugs its unknown since they are seldom notified and therefore not reported. Material and methods: retrospective analysis of the files of the Tuberculosis Clinic at the Hospital General Tijuana to determine the frequency of AR in patients treated with a combination of first and second line antituberculosis drugs. Results: The most frequent AR to antituberculosis drugs were: renal dysfunction (75.9\%), hepatitis (82.8\%), hematologic abnormalities (79.3\%), digestive AR (40\%), hearing loss (26.7\%), vestibular symptoms (15\%) and tendinopathy (30\%). Conclusions: AR to antituberculosis drugs are common and contribute to noncompliance. They must be routinely monitored and deal with, either with ancillary drugs, drug schedule modification and if needed with cessation of the offending drug.

Key words: Tuberculosis, drugs, adverse reactions, toxicity.

\section{INTRODUCCIÓN}

Los pacientes que reciben tratamiento para tuberculosis pansensible exclusivamente con fármacos de primera línea, suelen mostrar una excelente tolerancia al régimen de tratamiento y de presentarse efectos secundarios, éstos suelen ser leves y transitorios., ${ }^{1,2}$ Empero, los regímenes de tratamiento requeridos en la tuberculosis resistente a fármacos (TB-FR) incluyen medicamentos que suelen provocar con regularidad efectos adversos (EA), que en ocasiones requieren incluso la interrupción de un fármaco en particular. ${ }^{3}$

La verdadera frecuencia de EA atribuibles al tratamiento de la TB-FR es desconocida ya que por lo común no se notifican y, por ello, no se reportan. Sin embargo, en la práctica diaria es obvio que la mayoría de los pacientes presentan EA que complican el curso del tratamiento. Se han descrito en la literatura tasas de EA hasta en el $80 \%$ de los pacientes sometidos a tratamiento para tuberculosis multifarmacorresistente (TB-MFR), y la necesidad de modificar el régimen en más de la mitad de los casos. ${ }^{4}$

Un problema asociado a los esquemas con múltiples drogas utilizadas en el tratamiento de la TB-RF, es el hecho de que varios de estos fármacos comparten efectos adversos y toxicidad, lo que dificulta determinar cuál de ellos puede ser el responsable. Aunque por fortuna la aparición de complicaciones graves es infrecuente, los medicamentos antituberculosos tienen el potencial de generar toxicidad muy severa y potencialmente fatal. Esto incluye la anafilaxia y reacciones tóxicas como dermatitis exfoliativa y síndrome de Stevens-Johnson, 
gastritis erosiva con sangrado de tubo digestivo, hepatitis fulminante y falla renal. ${ }^{3}$

Con el propósito de determinar la asiduidad de los EA en pacientes con tuberculosis sometidos a esquemas de tratamiento mixtos con fármacos de primera y segunda línea, se llevó a cabo una revisión de los archivos de la Clínica de Tuberculosis Resistente a Fármacos del Hospital General Tijuana.

\section{PACIENTES Y MÉTODOS}

La Clínica y Laboratorio de Tuberculosis del Hospital General Tijuana (México) constituye un centro de referencia regional para pacientes con TB-RF, tuberculosis en situaciones especiales (p. ej. embarazo, casos pediátricos, etc.) y reacciones adversas a fármacos antituberculosis (RAFA). Se realizó una revisión de los expedientes clínicos del 01 de junio de 2012 al 31 de diciembre de 2014 con el fin de determinar la presencia de RAFA asociadas al tratamiento.

El protocolo de monitoreo rutinario de los pacientes incluye además de la evaluación clínica, exámenes mensuales de seguridad (biometría hemática, perfil bioquímico), evaluación de la agudeza visual mensual (tabla de Snellen, láminas de Ishihara), pruebas bimensuales de funcionamiento tiroideo en pacientes que reciben etionamida o protionamida y/o ácido Paminosalicílico (PAS), así como audiometría bimensual.

Los esquemas utilizados varían según el caso incluyen medicamentos de primera línea en casos pansensibles referidos por RAFA, y fármacos de primera y segunda línea en casos con resistencia a fármacos (tabla 1). Los medicamentos orales se administran de lunes a sábado y los inyectables de lunes a viernes, espaciándolos habitualmente a tres veces por semana al convertir el cultivo.

Para fines de esta revisión definimos las alteraciones fisiológicas como:
1. Alteración de la función renal:

a. $B U N>18 \mathrm{mg} / \mathrm{dL}$, creatinina sérica $>1.3 \mathrm{mg} / \mathrm{dL}$

2. Alteración de la función hepática:

a. Alanino aminotransferasa (ALT) y aspartato aminotransferasa (AST) $>3-5$ el límite normal superior $(40 \mathrm{UI} / \mathrm{L}),{ }^{5}$ bilirrubina total $>1.3 \mathrm{mg} /$ $\mathrm{dL}$, bilirrubina indirecta $>0.9 \mathrm{mg} / \mathrm{dL}$ y bilirrubina directa $>0.4 \mathrm{mg} / \mathrm{dL}$

3. Alteración hematológica:

a. Hemoglobina $<13.6 \mathrm{~g} / \mathrm{dL}$, leucocitos $<4.3 \mathrm{y}$ plaquetas $<156,000$ por $\mu \mathrm{L}$

4. Hipotiroidismo: hormona estimulante del tiroides $(\mathrm{TSH})>4.0 \mathrm{mlU} / \mathrm{L}$

5. Afectación auditiva: una pérdida por debajo de 20 $\mathrm{dB}$ se consideró como indicadora de lesión auditiva.

Análisis estadístico: se llevó a cabo con el paquete comercial SPSS versión 19. En la comparación de proporciones se utilizó la prueba de $\chi^{2}$ (y exacta de Fisher para comparaciones con menos de cinco observaciones por celda).

\section{RESULTADOS}

Se incluyeron 60 pacientes que recibieron tratamiento con drogas de primera y segunda línea. La edad media del grupo fue de $31.5 \pm 15.1$ años (rango 15 a 72 años). Entre los antecedentes de importancia 33 (55.3\%) pacientes tenían historia de alcoholismo con duración de $2.74 \pm 6.4$ años; 17 (29.3\%) refirieron historia de tabaquismo (duración $4.7 \pm 1.0$ años). 15 pacientes (25.9\%) refirieron diversos tipos de adicciones a diversas drogas ilícitas, en especial metanfetaminas por un espacio de $0.47 \pm 1.6$ años.

Nueve pacientes presentaron monorresistencia (15\%), siendo las más comunes la resistencia a estreptomicina y a isoniacida; 20 pacientes (33.3\%) manifestaron polirresistencia (siendo la más común la

Tabla 1. Esquemas de tratamiento utilizados en pacientes con mono, polirresistencia y multidrogorresistencia.

\begin{tabular}{ccc}
\hline Resistencia a & Tratamiento & Duración mínima (meses) \\
\hline H ( \pm S) & R, Z, E y fluoroquinolona & 9 \\
H y Z & R, E y fluoroquinolona & 12 \\
H y E & R, Z y fluoroquinolona & 12 \\
H, E y Z & R, fluoroquinolona + fármaco oral de segunda & 18 \\
& línea + inyectable de segunda línea durante los & 18 \\
\hline
\end{tabular}

$\mathrm{H}$ : isoniacida; Z: pirazinamida; E: etambutol; R: rifampicina.

*Antes de que la OMS instituyera esta directiva los pacientes con monorresistencia a rifampicina se trataban con $\mathrm{H}$, $\mathrm{E}$, fluoroquinolonas y un inyectable de segunda línea por 2-3 meses. 
resistencia a isoniacida y estreptomicina); y 28 (46.7\%) presentaron una cepa multifarmacorresistente. Los tres pacientes restantes fueron referidos precisamente por una RAFA. 32 pacientes (54.2\%) habían sido tratados con fármacos antituberculosis en el pasado (1.45 \pm 0.7 tratamientos); 6 pacientes (19.4\%) habían recibido tratamiento en dos ocasiones previas, y 4 pacientes (12.9\%) fueron tratados en el pasado en tres ocasiones. Sólo 2 pacientes $(3.4 \%)$ habían sido tratados con fármacos antituberculosis de segunda línea en el pasado.

Nueve pacientes (15.3\%) fueron referidos por presentar enfermedad por una cepa de $M$. tuberculosis monorresistente, $20(33.9 \%)$ por polirresistencia y 28 (47.5\%) por TB-MFR. Tres pacientes habían sido referidos por presentar RAFA de primera línea. La tabla 2 muestra la relación de fármacos utilizados, a menudo una combinación de fármacos de primera y segunda línea.

\section{RAFA asociadas a la función renal}

Los pacientes (casi de manera exclusiva con TB-MFR) recibieron períodos prolongados de inyectables por conversión tardía del cultivo. La kanamicina $(\mathrm{Km})$ se administró por $7.0 \pm 1$ meses, la amikacina $(\mathrm{Am})$ por $7.15 \pm 1.7$ y la capreomicina $(\mathrm{Cm})$ por $7.4 \pm 2.1$ meses.

Cuarenta y cuatro pacientes $(75.9 \%)$ presentaron alteración de la función renal; 35 (60.3\%) elevación del BUN $(26.1 \pm 1.6 \mathrm{mg} / \mathrm{dL})$ y 21 elevación de la creatinina sérica $(1.49 \pm 0.3 \mathrm{mg} / \mathrm{dL})$. La conducta ante la elevación de los azoados en pacientes que aún no lograban la conversión bacteriológica consistió en espaciar el inyectable a tres veces por semana, y de no haber mejoría

Tabla 2. Fármacos antituberculosis de primera y segunda línea incluidos en los regímenes de tratamiento.

\begin{tabular}{lcc}
\hline \multicolumn{1}{c}{ Fármaco } & Número & (\%) \\
\hline Isoniacida (H) & 21 & 35 \\
Rifampicina (R) & 33 & 55 \\
Etambutol (E) & 47 & 78.3 \\
Pirazinamida (Z) & 30 & 50 \\
Kanamicina (Km) & 3 & 5 \\
Amikacina (Am) & 27 & 45 \\
Capreomicina (Cm) & 18 & 30 \\
Levofloxacino (Lfx) & 45 & 75 \\
Moxifloxacino (Mfx) & 26 & 43.3 \\
Etionamida (Eto) & 10 & 16.7 \\
Protionamida (Pto) & 20 & 33.3 \\
Cicloserina (Cs) & 30 & 50 \\
Ácido P-aminosalicílico (PAS) & 16 & 26.7 \\
Linezolid (Lzd) & 3 & 5 \\
Claritromicina (Clr) & 2 & 2.3 \\
\hline
\end{tabular}

suspender en forma definitiva el inyectable. Una paciente $(1.7 \%)$ con diabetes de larga evolución progresó a insuficiencia renal, normalizándose los parámetros en el resto al suspender el inyectable.

\section{RAFA asociadas a la función hepática}

Cuarenta y ocho pacientes (82.8\%) manifestaron alteración de la función hepática. $30(50 \%)$ presentaron elevación de la AST (150 $\pm 47.8 \mathrm{UI} / \mathrm{L})$; en un paciente la AST se elevó a 800 UI/L. 29 pacientes (48.3\%) mostraron elevación de la ALT $(210.4 \pm 40.9$ UI/L). El mismo paciente con elevación máxima de AST presentó una determinación de ALT de 1,222 UI/L.

Quince pacientes $(25.9 \%)$ exhibieron elevación de la bilirrubina total $(2.64 \pm 0.7 \mathrm{mg} / \mathrm{dL})$; el paciente con elevación máxima de transaminasas tuvo una determinación máxima de bilirrubina total de $15.7 \mathrm{mg} / \mathrm{dL}$, siendo el único paciente con ictericia.

Si la condición clínica del caso lo permitía se suspendió el tratamiento hasta que las enzimas hepáticas se encontraran al menos $<3$ veces el límite superior normal. En caso de que el esquema inicial hubiera incluido pirazinamida, este fármaco se suspendía de manera definitiva y se elaboraba un esquema con fármacos no hepatotóxicos (fluoroquinolonas, cicloserina, etambutol, inyectables de segunda línea, linezolid y de no existir resistencia se intentaba reintroducir la rifampicina).

\section{RAFA asociadas a alteraciones hematológicas}

Cuarenta y seis pacientes $(79.3 \%)$ mostraron alteraciones hematológicas. $42(70 \%)$ anemia leve (11.5 \pm $2.3 \mathrm{~g} / \mathrm{dL}$ ) con un mínimo de $8 \mathrm{~g} / \mathrm{dL}$. Este paciente se encontraba recibiendo linezolid y la anemia se trató con eritropoyetina. 31 pacientes $(53 \%)$ manifestaron leucopenia (mínimo 3,700 mm³). 28 pacientes (48.3\%) presentaron trombocitopenia (mínimo $71,000 \mu \mathrm{L}$ ). Después de interconsultar a hematología no fue necesario suspender el tratamiento en ningún caso.

\section{RAFA asociadas al sistema digestivo}

Veintiún (35\%) pacientes tuvieron náusea y 14 vómito $(23.3 \%)$ que requirieron tratamiento sintomático (meclizina 30 minutos antes de la dosis de los fármacos antituberculosis más omeprazol por las noches). Cinco pacientes $(8.3 \%)$, todos ellos recibiendo PAS presentaron diarrea, que ameritó la suspensión del fármaco. Tres pacientes (5\%) refirieron dolor cólico abdominal y 4 anorexia $(6.7 \%)$. 


\section{RAFA asociadas a daño del VIII par craneal}

Dieciséis pacientes (26.7\%) que recibían inyectables de segunda línea presentaron disminución de la agudeza auditiva en comparación con la línea basal, demostrada por audiometría. Tres pacientes $(5 \%)$ refirieron acúfenos, $3(5 \%)$ ataxia y $3(5 \%)$ vértigo. En los pacientes con ataxia o vértigo se suspendió el inyectable de inmediato.

\section{RAFA asociadas a agudeza visual}

Tres pacientes (5\%) que recibían etambutol exhibieron disminución de la agudeza visual en comparación con la evaluación basal. Se suspendió el fármaco de inmediato.

\section{RAFA asociadas a manifestaciones dermatológicas}

Cuatro pacientes (6.7\%) presentaron prurito, 4 (6.7\%) urticaria y uno (1.7\%) desarrolló síndrome de StevensJohnson. En todos ellos se suspendió el esquema y se reintrodujeron poco a poco los fármacos para determinar el agente causal. En el caso de Stevens-Johnson se substituyó el esquema primario por fármacos de segunda línea para evitar el riesgo de una recaída.

\section{RAFA asociadas al sistema musculoesquelético}

Tres de los 71 pacientes que fueron tratados con una quinolona $(4.2 \%)$ presentaron mialgias, 10 pacientes $(14.1 \%)$ refirieron artralgias y en $5(7.0 \%)$ se diagnosticó tendinitis; ningún paciente tratado sin quinolonas presentó RAFA musculoesquelética $(p=0.08)$. No fue necesario interrumpir el fármaco en ningún caso.

\section{Otras RAFA}

Veinte pacientes presentaron como comorbilidad diabetes; 3 de ellos (15\%) desarrollaron polineuritis. Ninguno de los pacientes sin diabetes desarrolló polineuritis $(p=$ 0.03). Dos pacientes (3.3\%) refirieron insomnio, $3(5 \%)$ presentaron síndrome depresivo y un paciente $(1.7 \%)$ presentó un brote psicótico, todos ellos bajo tratamiento con cicloserina a dosis de $250 \mathrm{mg}$ dos veces al día. No se observaron estas RAFA en pacientes tratados con regímenes sin cicloserina $(p=0.02)$. Las RAFA cedieron con la suspensión del medicamento.

Veinte pacientes (33.3\%) con drogas de segunda línea presentaron RAFA lo suficientemente severas para ameritar modificación o suspensión de los medi- camentos. Las pruebas de función tiroidea bimensuales en pacientes tratados con etionamida, protionamida o PAS revelaron hipotiroidismo en 8 pacientes (17.4\%) que requirió tratamiento con levotiroxina. Ninguno de los pacientes con regímenes que no incluían estas drogas presentó hipotiroidismo $(\mathrm{p}=0.14)$.

En la cohorte analizada no se presentaron decesos atribuibles a la enfermedad o la presencia de RAFA.

\section{DISCUSIÓN}

Más del $80 \%$ de los pacientes incluidos en esta revisión presentaron algún tipo de EA, sobre todo alteraciones de la función renal y hepática; y una tercera parte de los pacientes que recibían drogas de segunda línea manifestaron RAFA, lo suficientemente severas para ameritar modificación o suspensión de los medicamentos.

La intolerancia a los fármacos antituberculosis debido a EA constituye un problema importante durante el tratamiento de este padecimiento. ${ }^{4}$ La falta de atención por parte del personal de salud a los EA puede tener serias implicaciones sobre la percepción del paciente acerca de la toxicidad vs. el beneficio del tratamiento y puede, luego entonces, impactar la adherencia al mismo. ${ }^{6}$ Se debe prestar particular atención a los EA reportados por el paciente; la mayoría de los enfermos están dispuestos a continuar con el régimen a pesar de las molestias cuando entienden el beneficio del mismo, y de informarles que en la mayoría de los casos las molestias disminuyen después de las primeras semanas. ${ }^{7}$

El daño hepático inducido por fármacos antituberculosis es uno de los EA más comúnmente asociados al tratamiento de la tuberculosis. Este EA disminuye la efectividad del régimen al favorecer la falta de adherencia pudiendo llevar al fracaso terapéutico y a la recurrencia o emergencia de resistencia a fármacos. Algunos polimorfismos de las enzimas hepáticas se han asociado a daño hepático por fármacos antituberculosis (p. ej. acetiladores lentos). La presencia de coinfección viral por el virus de la inmunodeficiencia humana $(\mathrm{VIH})$ y de hepatitis $\mathrm{C}$ también se asocia a mayor frecuencia de daño hepático por fármacos antituberculosis. El riesgo relativo de pacientes coinfectados por hepatitis $C$ es cinco veces mayor, cuatro veces mayor para coinfectados con VIH y 14.4 veces mayor para los coinfectados con hepatitis $\mathrm{C}$ y $\mathrm{VIH}^{8}$

Los aminoglucósidos constituyen uno de los factores de riesgo más comunes para nefrotoxicidad. Ésta se manifiesta clínicamente como insuficiencia renal no oligúrica con un incremento progresivo de la creatinina sérica y diuresis hiposmolar. Los aminoglucósidos son nefrotóxicos debido a que una proporción de la dosis administrada $(-5 \%)$ se retiene en las células 
epiteliales de los túbulos proximales. ${ }^{9}$ La ototoxicidad de los aminoglucósidos se reconoció hace más de 70 años a raíz de la aplicación inicial de la estreptomicina (SM). La toxicidad coclear de los aminoglucósidos se manifiesta como una pérdida bilateral irreversible de la agudeza auditiva, iniciando por las frecuencias altas; la toxicidad vestibular se manifiesta por vértigo, náusea, vómito, nistagmo y ataxia. La severidad potencial del daño depende del aminoglucósido utilizado. La km se considera más ototóxica que la Am. La Am afecta más la rama vestibular del octavo par, mientras que la estreptomicina afecta más el epitelio vestibular. ${ }^{10}$

La tendinitis y ruptura tendinosa se han reportado en asociación al uso de la mayoría de las fluoroquinolonas. La mayoría de las lesiones se presentan en el tendón de Aquiles, típicamente alrededor de las dos semanas de tratamiento, pero pueden pasar meses antes de que esto ocurra. Los factores asociados con la tendinitis por fluoroquinolonas incluyen la nefropatía (quizás relacionada con el aclaramiento del fármaco), la edad avanzada, la diabetes mellitus, las enfermedades reumáticas y el uso crónico de esteroides sistémicos. ${ }^{11}$

Cuando se presenta un EA durante el tratamiento, como primera medida hay que verificar que las dosis de los fármacos sean las apropiadas para el peso, edad y características especiales del paciente (p. ej. presencia de nefropatía o hepatopatía previa). Es necesario descartar que la sintomatología se deba a otra causa y no a los fármacos (p. ej., en el caso de hepatitis que ésta pudiese tener un origen viral). ${ }^{7}$

Es fundamental informar al paciente sobre estos efectos y la mínima posibilidad de cambio del esquema para lograr su colaboración y no verse en la necesidad de suspender el tratamiento. Se discutirán con el paciente los beneficios y riesgos del tratamiento antes de iniciar el régimen y la necesidad de incluir múltiples fármacos para poder integrar un régimen efectivo. El paciente debe comprender la importancia de cada uno de los fármacos incluidos en el esquema y sus efectos adversos y toxicidad. Hay que asegurarle al paciente que se harán todos los esfuerzos para reducir al máximo las molestias asociadas al tratamiento, pero que deberá estar mentalmente preparado para continuar con el régimen a pesar de los EA. El paciente debe estar consciente de que ésta puede ser su última oportunidad de curación y que de abandonar el presente tratamiento, otros futuros regímenes, si es que es posible estructurar alguno, serán aún más tóxicos y menos efectivos. Es indispensable reducir al máximo las interrupciones en el tratamiento debido a EA pues esto reduce la efectividad de los fármacos y puede favorecer la amplificación de la resistencia ya existente. ${ }^{12}$
En el caso de efectos gastrointestinales (náusea y/o vómito), el fraccionar las dosis en un par de tomas en el día puede mejorar la tolerancia a estos fármacos. Se puede utilizar un antiemético (clorpromazina, ondansetron, domperidona, meclizina, etc.) o un procinético ( $p$. ej. metoclopramida) 30 minutos antes de administrar la dosis del medicamento y después por horario si se requiere; el uso de antiácidos o sucralfato debe evitarse en las dos horas previas y posteriores a la administración de las fluorquinolonas pues interfieren con su absorción. Se puede intentar que el paciente no reciba los medicamentos en ayuno al permitir que coma unas galletas o pan tostado antes del medicamento. Como profilaxis para neuropatía periférica se recomienda agregar $100 \mathrm{mg}$ diarios de piridoxina (B6) al régimen en todos los pacientes con TB-MFR cuyo esquema incluya etionamida, cicloserina, linezolid o isoniacida a dosis altas. ${ }^{13}$

Una limitación de nuestro estudio, como ocurre en cualquier estudio retrospectivo, es que somos conscientes de que quizás no se hayan documentado todos los EA en todos los casos; en especial, si éstos eran leves o transitorios y que las tasas reportadas sean una subestimación de la verdadera frecuencia con que se presentan las RAFA, en especial en pacientes tratados con fármacos de segunda línea.

\section{CONCLUSIÓN}

Los EA de los fármacos antituberculosis sobre todo las drogas de segunda línea son muy frecuentes, y constituyen un factor muy importante en la pérdida de adherencia al régimen. Deben ser monitoreados activamente durante el seguimiento clínico, y mitigados con tratamiento sintomático, cambios en los horarios de administración y si el caso lo amerita, la suspensión del fármaco en cuestión.

\section{REFERENCIAS}

1. Törün T, Güngör G, Özmen I, et al. Side effects associated with the treatment of multidrug-resistant tuberculosis. Int J Tuberc Lung Dis 2005;9(12):1373-1377.

2. Arbex MA, Varella M de C, Siqueira HR, Mello FA. Antituberculosis drugs: drug interactions, adverse effects, and use in special situations. Part 1: First-line drugs. J Bras Pneumol 2010;36(5):626-640.

3. Arbex MA, Varella M de C, Siqueira HR, Mello FA. Antituberculosis drugs: drug interactions, adverse effects, and use in special situations. Part 2: Second-line drugs. J Bras Pneumol 2010;36(5):641-656.

4. Farazi A, Sofian M, Jabbariasl M, Keshavarz S. Adverse reactions to antituberculosis drugs in Iranian tubercu- 
losis patients. Tuberc Res Treat 2014;2014:412893. doi: $10.1155 / 2014 / 412893$.

5. Saukkonen JJ, Cohn DL, Jasmer RM, et al.; ATS (American Thoracic Society) Hepatotoxicity of Antituberculosis Therapy Subcommittee. An official ATS statement: hepatotoxicity of antituberculosis therapy. Am J Respir Crit Care Med 2006;174(8):935-952.

6. Shean K, Streicher E, Pieterson E, et al. Drug-associated adverse events and their relationship with outcomes in patients receiving treatment for extensively drug-resistant tuberculosis in South Africa. PLoS One 2013;8(5):e63057. doi: 10.1371/journal.pone.0063057.

7. Laniado-Laborín R. Adverse effects and toxicity of antituberculosis drugs. In: Drug resistant tuberculosis. Practical guide for clinical management. ISBN 978-168108-067-3. Bentham e-books: 2015. p. 74-84.

8. Shang P, Xia Y, Liu F, et al. Incidence, clinical features and impact on anti-tuberculosis treatment of anti-tuberculosis drug induced liver injury (ATLI) in China. PLoS One 2011;6(7):e21836. doi: 10.1371/journal.pone.0021836.

9. Mingeot-Leclercq MP, Tulkens PM. Aminoglycosides: nephrotoxicity. Antimicrob Agents Chemother 1999;43(5):1003-1012.

10. Xie J, Talaska AE, Schacht J. New developments in aminoglycoside therapy and ototoxicity. Hear Res 2011;281(12):28-37. doi: 10.1016/j.heares.2011.05.008.
11. Khaliq Y, Zhanel GG. Fluoroquinolone-associated tendinopathy: a critical review of the literature. Clin Infect Dis 2003;36(11):1404-1410.

12. Curry International Tuberculosis Center and California Department of Public Health. Adverse reactions. In: California Public Health Department, editors. DrugResistant Tuberculosis: A Survival Guide for Clinicians. 3rd ed. USA; 2016: p. 146-171.

13. Laniado-Laborín R. Efectos adversos y toxicidad de los fármacos antituberculosis. En: El ABC de la tuberculosis resistente a los fármacos: Manual práctico de diagnóstico y tratamiento. ISBN-10: 3848459884. Editorial Académica Española; 2012: p.78-92.

\section{$\triangle$ Correspondencia:}

Dr. Rafael Laniado-Laborín

Emiliano Zapata Núm. 1423,

Zona Centro, Tijuana Baja California.

Teléfono/fax: (664) 686-5626

Correo electrónico: rlaniado@uabc.edu.mx

Los autores declaran no tener conflicto de intereses. 